\title{
Quantitative Assessment of HER2 Gene Amplification of Breast Cancer Using Droplet Digital PCR
}

\section{Kazutaka Otsuji}

the University of Tokyo https://orcid.org/0000-0002-5688-5536

Takeshi Sasaki ( $\nabla$ takesasa@m.u-tokyo.ac.jp )

University of Tokyo https://orcid.org/0000-0001-8638-5412

Masahiko Tanabe

The University of Tokyo

Yasuyuki Seto

The University of Tokyo

Research article

Keywords: Breast cancer, HER2, gene amplification, droplet digital PCR (ddPCR), tumor content ratio (TCR), eHER2

Posted Date: December 3rd, 2020

DOI: https://doi.org/10.21203/rs.3.rs-116841/v1

License: (c) (i) This work is licensed under a Creative Commons Attribution 4.0 International License. Read Full License 


\section{Abstract}

We previously reported the usefulness of droplet digital polymerase chain reaction (ddPCR) for the assessment of Human epithelial growth factor receptor 2 (HER2) gene amplification in breast cancer using formalin-fixed and paraffin-embedded sections. In our previous study, we combined HER2/CEP17 ratio (HER2 gene signals to chromosome 17 signals) with ddPCR and tumor content ratio (TCR) of each sample and determined the HER2 status by adopting a two-dimensional chart. This "ddPCR-TCR method" showed a high concordance with conventional HER2 status. In this study, we updated our method to assess the HER2 status of breast cancer in a more quantitative manner. We combined obtained data of the ddPCR ratio $\left[\mathrm{R}_{x}\right]$ and TCR $[x]$; we calculated " $\left(\mathrm{R}_{x}-1\right) / x+1$ " for 41 samples with primary breast cancer and named the value led by this formula as "eHER2 (estimated HER2/CEP17 ratio of a tumor cell)". eHER2 was equivalent to conventional ISH HER2/CEP17 ratio in most cases. eHER2 and ISH ratio showed a very strong correlation (Spearman rank correlation; $\rho=0.70, P<0.0001$ ). The obtained results indicated that $e H E R 2$ is a potential tool for HER2 status diagnosis in breast cancer.

\section{Introduction}

Human epithelial growth factor receptor 2 (HER2) is a member of the epidermal growth factor receptor family [1]. HER2 gene amplification is the main mechanism of HER2 protein overexpression [2], and HER2 gene-amplified and/or HER2 protein-overexpressed cancer is determined as HER2-positive. HER2 is positive in $10 \%-25 \%$ of human breast cancers, and its positivity is associated with aggressive metastatic disease and a poor prognosis. However, since trastuzumab, an anti-HER2 antibody, became available in daily clinical practice, the prognosis of HER2-positive breast cancer has improved dramatically [1, 2]. At present, HER2 protein expression is generally assessed by immunohistochemistry (IHC). Although IHC is easy to perform, the results from different examinations can vary according to sample conditions or specific staining processes. Thus, it is often challenging to make consistent classification of HER2 status with IHC. HER2 gene copy number is detected using an in situ hybridization (ISH). ISH provides better diagnostic accuracy and added confidence, but it is more time-consuming, labor-intensive, and expensive compared to IHC.

To solve current problems of HER2 status diagnosis, we previously reported the usefulness of droplet digital polymerase chain reaction (ddPCR) for the assessment of HER2 gene amplification in breast cancer using formalin-fixed and paraffin-embedded (FFPE) sections [3]. ddPCR has gained attention as a more precise and less subjective method to quantify DNA amplification [4]. In our previous study, we calculated the HER2/CEP17 ratio (HER2 gene signals to chromosome 17 signals) with ddPCR and the tumor content ratio (TCR) with a digital slide scanner for each sample. The calculated values were plotted on a two-dimensional chart, named ddPCR-TCR chart: samples plotted above the cut-off line was determined as HER2 positive; those plotted below the line as HER2 negative. This method of HER2-status determination, named "ddPCR-TCR method," showed a high concordance with conventional approaches to evaluate HER2 status. Furthermore, we not only succeeded in automating a large part of the process 
from DNA extraction to determination of HER2 status but also reduced the costs compared with conventional HER2 examinations [3].

A limitation of our previous method was that the way of evaluating HER2 status was more qualitative than quantitative because of the necessity of a two-dimensional chart. In this study, we updated the ddPCR-TCR method intending to assess HER2 status in breast cancer in a more quantitative manner. We statistically analyzed the obtained data and examined whether our new strategy is applicable for clinical use.

\section{Methods}

\section{Patient cohort}

FFPE samples were collected from 41 primary breast cancer patients, the same cohort as our previous study [3], who underwent surgery at The University of Tokyo Hospital from 2009 to 2011 (Table 1). Patients with ductal carcinoma in situ, a tumor size of $<1 \mathrm{~cm}$, and history of preceding chemotherapies were excluded. The median age of the patients was 60.0 years (range, 28-85 years). Tumor sizes ranged from 10 to $43 \mathrm{~mm}$. All samples included in the study had been previously assessed for their HER2 status according to the previous version of American Society of Clinical Oncology/College of American Pathologists (ASCO/CAP) guidelines [5]. A tumor was considered positive for HER2 when the IHC scoring result was score $3+$ positive (circumferential membrane staining that is complete, intense, and in $>10 \%$ of tumor cells) or $2+$ (circumferential membrane staining that is incomplete and/or weak/moderate and in $>10 \%$ of tumor cells or complete and circumferential membrane staining that is intense and in $\leq 10 \%$ of tumor cells) with a HER2/CEP17 ratio of $\geq 2.0$ or average HER 2 gene copy of $\geq 6.0$ signals/cell determined using either fluorescence in situ hybridization (FISH) or differentiation induction subtraction hybridization (DISH), counting at least 20 cells within the area. A tumor was considered negative for HER2 when the IHC scoring result was score 0 (no membrane staining), $1+$ (faint or barely perceptible/incomplete membrane staining), or $2+$ with a HER2/CEP17 ratio of $<2.0$ and average HER2 gene copy of $<4.0$ signals/cell determined by ISH. This study had no ISH equivocal cases (HER2/CEP17 ratio: <2.0; average HER2 gene copy: $\geq 4.0$ and $<6.0$ signals/cell). Among 10 HER2 $3+$ cases, four cases were determined as HER2 $2+$ at the time of diagnosis according to firstly published guidelines [2]. Finally, 15 patients were diagnosed as positive for HER2.

\section{ddPCR and TCR calculation}

The data of ddPCR ratio of HER2/CEP17 and TCR were obtained in our previous report [3]. Briefly, DNA was extracted from FFPE sections of the samples and purified using the MagCore® Genomic DNA FFPE One-Step Kit following the manufacturer's instructions (Cartridge Code: 405; running time: 2 h; elution volume: $60 \mu \mathrm{L}$ ). Neither macro- nor micro-dissection were not performed before DNA extraction from FFPE samples. ddPCR was performed on a QX200 droplet digital PCR system (Bio-Rad) with HER2 primers (ERBB2-13F: CCCTCCGTACTTCCTGATGCT, ERBB2-13R: GCCATGGAGAGCCTCACATT, and ERBB2-13P: FAM/TGAGAGTCA/ZEN/AGATCTC/3IABkFQ) and CEP17 primers (ch17cent-6F: 
CGCTCCTGCACTGTAACACGT, ch17cent-6R: TCATTCCTGCAGCCCTTGA, and ch17cent-6P:

$\mathrm{VIC/AGCAGGTCC/ZEN/AGCCCA/3IABkFQ)} \mathrm{(Integrated} \mathrm{DNA} \mathrm{Technologies,} \mathrm{Coralville,} \mathrm{IA,} \mathrm{USA).} \mathrm{PCRs} \mathrm{were}$ performed in a total volume of $20 \mu \mathrm{L}$ containing $10 \mu \mathrm{L}$ Bio-Rad $2 \times$ ddPCR Supermix for Probes (no dUTP), HER2 primers (500 nM ERBB2-13F, 500 nM ERBB2-13R and 250 nM ERBB2-13P), CEP17 primers ( $500 \mathrm{nM}$ ch17cent-6F, $500 \mathrm{nM}$ ch17cent-6R and $250 \mathrm{nM}$ ch17cent-6P), 10-260 ng DNA, and water. The reaction mixtures were partitioned into an emulsion of approximately 20,000 droplets in oil using a QX200 Droplet Generator. After performing PCR, the PCR plate was loaded on a Bio-Rad QX200 droplet reader and read using Bio-Rad QuantaSoft version 1.6.6 software. The HER2/CEP17 copy number ratio was analyzed by calculating the copies per droplet from the Poisson distribution [6].

TCRs were calculated as the number of AE1/AE3-positive (1:200, Leica Biosystems) cells within the tumor region divided by the number of all detected cells in FFPE tissue sections. We used Definiens Tissue Studio (ver 3.6; Munich, Germany) to count stained and unstained cells separately [7-9].

Estimated HER2/CEP17ratio of a tumor cell (eHER2)

In our previous report [3], a two-dimensional "ddPCR-TCR chart" was used to determine HER2 gene amplification. In this chart, the vertical axis represents the ddPCR ratio $[R](0<R)$ and the horizontal axis represents the TCR $[X](0<X \leq 1)$. An FFPE section contains both tumor and non-tumor cells, and thus the extracted DNA is derived from both kinds of cells. If there are exactly twice as many HER2 genes as CEP17 in a tumor cell, the HER2/CEP17 ratio obtained by ddPCR [ $\left.\mathrm{R}_{x}\right]$ can be expressed as $x+1$ (Fig. 1a). If one of the samples is plotted above the theoretical cut-off line $x+1$, the cancer cells in this sample are considered to have HER2 gene amplification (HER2/CEP17 ratio over 2.0) (Fig. 1b). In determining the HER2 statuses of the clinical samples, instead of setting a clear cut-off line, we settled an equivocal area on the chart that we calculated in a cell line assay.

In the current study, we developed a new strategy to assess HER2 status in a more quantitative way by combining data of ddPCR ratio and TCR. Suppose there is a sample in which the HER2/CEP17 ratio in tumor cells $\left(\mathrm{R}_{1}\right)$ is unknown, but its ddPCR ratio $\left[\mathrm{R}_{x}\right]$ and TCR $[x]$ are successfully obtained; $\mathrm{R}_{x}$ can then be expressed as " $\left(\mathrm{R}_{1}-1\right) x+1$ ". Thus, $\mathrm{R}_{1}$, the unknown HER2/CEP17 ratio, can be calculated from the following formula: $\left(\mathrm{R}_{x}-1\right) / x+1(0<x \leq 1)$. We named this HER2/CEP17 ratio " $\mathrm{R}_{1}$ " calculated using ddPCR ratio $\left[\mathrm{R}_{x}\right]$ and TCR $[x]$ as "eHER2" (estimated HER2/CEP17 ratio of a tumor cell) (Fig. 1c).

Figure $1 \mathrm{~d}$ is a graphical concept of eHER2. The obtained TCR $(x)$ bears a proportionate relationship to $\left(\mathrm{R}_{x}\right.$ - 1), so $\left(R_{x}-1\right) / x$ is equal to $\left(R_{1}-1\right) / 1$. Hence eHER2 $\left(=R_{1}\right)$ can be expressed as $\left(R_{x}-1\right) / x+1$.

Alternatively, $\operatorname{eHER2}\left(=\mathrm{R}_{1}\right)$ can be calculated by plugging " $X=1$ " into ${ }^{R}=\frac{R x-1}{x} X+1$, a straight line connecting two points, $(X, R)=(0,1)$ and $\left(X, \mathrm{R}_{X}\right)$, on the graph. In the current study, eHER2 was calculated for all the samples, and these values were compared to the obtained conventional ISH ratios.

\section{Setting equivocal range}


In establishing the ddPCR-TCR method in our previous report [3], we set the equivocal area on the chart that we obtained by cell line assay. In this assay, we extracted genomic DNA from two cell lines: one cell line assuming HER2 gene double-amplified tumor cells, and another cell line assuming HER2 nonamplified normal cells. We mixed the DNA in a stepwise manner to construct TCR patterns from 10-90\%, analyzed the samples using ddPCR three times, and plotted the results on a ddPCR-TCR chart.

In the assay, the linear regression line obtained from the plotted data was very close to our supposed cutoff line " $R=X+1$ ", which successfully confirmed our theory of the ddPCR-TCR method. However, we noticed that there were measuring errors using ddPCR analysis, even though it was a very small range. We assumed these errors were due to the manual process of preparing reagent solutions before analyzing gene amplification by ddPCR. These observations suggest that if we analyze the HER2/CEP17 ratio by ddPCR and calculate TCR of a sample containing tumor cells with double-amplified HER2 gene, the eHER2 might not be exactly 2.0 every time. The previous and ongoing ASCO/CAP guideline $[5,10]$ sets ISH positivity as HER2/CEP17 ratio $\geq 2.0$, so the cut-off value of $e H E R 2$ may theoretically be 2.0. However, determining cases with eHER2 only slightly less than 2.0 as "HER2 negative" would be risky.

To increase the sensitivity, we adopted the range of 1.8-2.2 as an equivocal range of eHER2 (Fig. 2a), based on the equivocal range for FISH assays of HER2 gene amplification in the firstly published version of ASCO/CAP guidelines [2]. This means that a sample with eHER2 between the range 1.8 to 2.2, or whose $\mathrm{R}_{x}$ is between $0.8 x+1$ and $1.2 x+1$, has the possibility of either HER2 gene twice-amplified and not.

However, there is another problem that also may decrease the accuracy of eHER2. When the TCR of a sample is high enough, the effect of the measuring error of ddPCR analysis on eHER2 calculation is not so large. In contrast, samples with very low TCR could result in an overestimated or underestimated eHER2. To overcome this problem, we set one more equivocal range to try to increase the specificity of our method. We rotated the equivocal area in Fig. 2a and fit it on ddPCR-TCR chart (Fig. 2b). This second equivocal area indicates that the determination of HER2 status by calculating eHER 2 can be misleading if a sample is plotted in this area, or in other words, if $R_{x}$ of the sample is between $1.2 x+0.8$ and $0.8 x+1.2$.

When applying these equivocal areas on the ddPCR-TCR chart with the clinical cases plotted [3], these areas almost overlap the previous equivocal area. Also, cases within the previous equivocal area (cases 3 , $4,8,22,27,32,33$ and 35 on Table 2) are all included in the newly combined equivocal areas (Fig. 2c).

\section{Statistical analysis}

Statistical analysis was performed using JMP Pro statistical software (ver. 12.2.0, SAS Institute, Japan). A non-parametric Spearman rank order correlation was used to determine the relationship between ISH ratio and $e H E R 2$. Receiver Operating Characteristic (ROC) curve was generated to evaluate the ability of eHER2 to discriminate HER2 positive case versus HER2 negative case.

\section{Results}


The data of conventional HER2 status (IHC score and ISH ratio), ddPCR ratio, TCR, and eHER2 for all 41 breast cancer samples are summarized in Table 2.

Correlation between HER2 IHC score and eHER2, ISH ration and eHER2, and HER2 status according to ASCO 2013 [5] and eHER2 are shown in Fig. 2a, b and c. There were significant differences for eHER2 between IHC 0,1 1 to $2+(P=0.040), 2+$ to $3+(P=0.003)$, and $0,1+$ to $3+(P=0.0016)$ (Fig. 3a). Also, eHER2 was significantly higher in ISH ratio $\geq 2.0$ cases compared to ISH ratio $<2.0$ cases $(P<0.001)$ (Fig. 3b). When diagnosed according to ASCO 2013 guidelines [5], HER2 positive cases showed significant higher $e H E R 2$ than HER2 negative cases $(\mathrm{P}<0.001)$ (Fig. $3 \mathrm{c})$. ISH ratio and $e H E R 2$ showed a very strong correlation (Spearman rank correlation; $\rho=0.70, P<0.0001$ ).

According to the ROC curve analysis, a cut-off value $=1.67$ of eHER2 was determined (sensitivity and specificity, $100 \%$ and $88 \%$, respectively, $P<0.0001$ ), and area under the curve was 0.982 (Fig. $3 d$ ).

\section{HER2 /CEP17 ratio of ISH and eHER2 for each sample}

Figure 4 shows the HER2/CEP17 ratio of ISH (blue bars) and eHER2 determinations (orange bars) for each sample. The theoretical cut-off value of 2.0 is depicted as dotted line, and one of the equivocal ranges 1.8-2.2 is expressed as a gray zone on the chart. Cases within either or both of the two newly established equivocal ranges are marked with asterisks, and their case numbers (Table 2) are indicated on the chart. All cases with an ISH ratio $\geq 2.0$ showed a high $e H E R 2$, at least over 1.8 . There were two discrepant cases (cases 4 and 36) in which the ISH ratio was less than 1.8 but eHER2 was over 2.2. Of these, case 4 was included within equivocal ranges, but case 36 was out of the ranges.

\section{Discussion}

In this study, we reaffirmed the potential of the usefulness of ddPCR for the assessment of HER2 gene amplification in breast cancer.

We developed the ddPCR-TCR method in our previous report and showed that HER2 gene amplification assessing in breast cancer using ddPCR was feasible for clinical use [3]. However, in ddPCR-TCR method, the determination of HER2 gene amplification in a tumor was based on the area on the ddPCR-TCR chart on which the case was plotted, making the assessment more qualitative than quantitative.

In the current study, we improved our method by applying a very simple but potent strategy to quantitatively assess HER2 status at the final step of the ddPCR-TCR method. We calculated the unknown ratio of HER2 gene to CEP17 in only tumor cells by combining the ddPCR ratio [R] and TCR [X] and named this calculated HER2/CEP17 ratio of tumor cells as eHER2. This new approach provides us information on HER2 gene amplification of breast cancer specimens using a very simple and costeffective method with ddPCR. 
Our method has several advantages compared with the standard evaluation techniques. IHC and ISH are subjective in determining HER2 status, and special training is necessary for professional pathologists to make an accurate judgment. However, even pathology specialists often have difficulty in judging some equivocal or borderline cases. Our method can obtain accurate and precise data of HER2 gene amplification of a tumor objectively and rapidly, which means that our method can relieve pathologists of a great burden. ddPCR is easy to perform, with only a few manual processes, and the running cost is far cheaper than ISH analyses. ISH is routinely performed for breast cancer specimens in some countries, but not all areas, mainly because of economic reasons. Our method using ddPCR provide information of HER2 gene amplification for breast cancer cases with very low costs, which would help reduce medical spending.

The calculation of $e H E R 2$ is the ultimate tool in our method. eHER2 is the estimated HER2/CEP17 ratio of tumor cells using the obtained data of ddPCR ratio and TCR. In this study, we showed a high correlation between ISH ratio and $e H E R 2$, and our results indicated that $e H E R 2$ is equivalent to ISH ratio. With our method, clinicians and pathologists will be able to obtain the data for the HER2/CEP17 ratio in breast cancers easily and cheaply without the technical needs or costs of performing ISH.

Although ddPCR is a very precise device for DNA amplification measurement, this method has a measuring error to some extent, and this makes it difficult to determine the HER2 status of cases with eHER2 only slightly higher or lower than 2.0. To evaluate such cases with caution, we set the equivocal range as $1.8 \leq e H E R 2 \leq 2.2$, or $0.8 x+1 \leq R_{x} \leq 1.2 x+1$, based on the determination of $H E R 2$ gene amplification with ISH in the first ASCO/CAP guidelines for breast cancer [2]. One factor that hinders the accuracy ddPCR analysis is the heterogeneity of HER2 expression and/or HER2 gene amplification within a tumor. Wang et al. evaluated HER2 gene amplification with ddPCR, specifically for the HER2 equivocal cases, and showed high accuracy and usefulness of ddPCR. Still, they also considered that the presence of intratumoral heterogeneity of HER2 would make the evaluation challenging [11]. Therefore, it seems reasonable to set the equivocal range in our method. Such cases within this range may well be determined their HER2 status comprehensively considering the results of conventional tools such as IHC and ISH. However, we speculate that this range could be set much narrower, considering the potential of ddPCR.

The effect of the error also depends on the TCR of each case; the influence of error is relatively small if TCR of a specimen is high enough, while range of the error of calculated $e H E R 2$ can be larger as TCR gets smaller. Based on this observation, we set another equivocal range: $1.2 x+0.8 \leq \mathrm{R}_{x} \leq 0.8 x+1.2$. This second equivocal range is graphically symmetrical about a point with the first equivocal range (Fig. 2). The $e H E R 2$ of cases within the second equivocal range can be calculated as far away from the true HER2/CEP17 of tumor cells, so we need to be careful when determining the HER2 status of a case. The eHER2 of cases with very low TCR but out of the second equivocal range may also be far from the true HER2/CEP17 but is still useful in HER2 status determination. For example, a case with relatively low TCR (e.g. 0.2 ) but with very high ddPCR ratio (e.g. over 2.0) would be HER2 positive without doubt, and vice versa. 
By setting these two equivocal ranges, we succeeded in improving sensitivity and specificity of our method. The ranges were quite similar to the equivocal area on ddPCR-TCR method in our previous study [3], and eight cases within the previous equivocal area were also included in our new equivocal ranges. One of the goals of our method is to accurately select HER2-positive cases and thus to determine appropriate candidates of anti-HER2 therapy. To increase the precision of our method, we need to determine more optical equivocal ranges in the future.

Most of the cases, except for cases within the equivocal ranges, showed strong concordance between conventional ISH ratio and our established eHER2, however one case (case number 36 in Table 2 and Fig. 4) showed discrepant results between the two. In this case, the ISH ratio was lower than 1.8 while eHER2 was higher than 2.2. HER2 gene amplification was calculated with FISH at the time of diagnosis and no longer observable because of degraded fluorescence, so we re-examined this sample with DISH. Five slides were granted to evaluate the quality of the DISH exam, and we found instability of CEP17 signals among the slides, indicating that the quality of FFPE specimen may be deficit owing to insufficient fixation or degradation of the tissue. The re-examined result of the HER2/CEP17 ratio with DISH was 1.56, which was similar to the value using FISH (1.70), but these ratios may be underestimated. The IHC staining results from this case also showed some discrepancies. One pathologist diagnosed this case as HER2 with an IHC score 3+, because complete circumferential membrane staining could be seen for at least $10 \%$ of tumor cells, but another pathologist diagnosed as HER2 score of $2+$ since membrane staining was not sufficient to determine as completely circumferential. These discrepant diagnoses of IHC between the two pathologists are likely owing to the low quality of the specimen. With our method using ddPCR, HER2/CEP17 ratio can be measured accurately unless the DNA of tumor cells is severely damaged, and at this point our method is still advantageous over conventional assays. Clinically, this patient did not receive anti-HER2 therapy after surgery, but fortunately no sign of recurrence has been seen over these six years.

Our method represents a novel strategy to evaluate HER2 status. Although this technique may be unusual for pathologists, who are trained to determine HER2 status by observing specimen, clinicians can easily integrate $e H E R 2$ into daily medical practice, as are trained in examinations that yield numerical values and ranges, such as blood tests. However, while IHC and ISH can be visually observed repeatedly and are preserved with prepared slides, the calculated ddPCR ratio, TCR and eHER2 are only calculated data, and these data must be managed carefully to avoid problems, such as patient mix-ups.

In this study, we focused on quantifying HER2 status determination and established the calculated value of $e H E R 2$, which has the potential to replace conventional HER2 examinations. One of the purposes of quantification in determining HER2 status is to simplify the evaluation for clinicians and pathologists to recognize the degree of HER2 gene amplification. However, our final goal is to use eHER2 as predictive factor of anti-HER2 therapy and a prognostic factor. Xu et al. evaluated the association between diseasefree survival and HER2 amplification level by ISH in a meta-analysis but concluded that HER2 amplification level is not a prognostic factor for HER2-positive breast cancer with trastuzumab-based targeted therapy [12]. We could not find a large-scale study that indicates the relationship between the 
rate of HER2/CEP17 with ISH and the effectiveness of anti-HER2 antibody. Also, there has been no report showing the relationship between HER2 status examined with ddPCR and the therapeutic effect of antiHER2 therapy in breast cancer, including the assessment of pathological complete response rates after preoperative chemotherapy. The direct target of currently marketed anti-HER2 drugs is indeed overexpressed-HER2 protein, not amplified-HER2 gene. However, ASCO/CAP guidelines $[2,5,10]$ treat IHC and ISH equally as methods for HER2 test in breast cancer; thus, we developed a technique to digitally assess HER2 gene amplification as a novel HER2 testing tool, which makes better use of the characteristics of ddPCR. In the future, it would be necessary to select cases that are preferable to receive intensive anti-HER2 therapy, e.g. trastuzumab plus pertuzumab, in the adjuvant setting for early HER2positive breast cancer, and $e H E R 2$ may play an important role in these cases. Additional studies including more cases are required, and future research should analyze whether $e H E R 2$ is a really useful predictive or prognostic marker for HER2-positive breast cancer patients.

In conclusion, we evolved the strategy for the ddPCR-TCR method from our previous report and proposed eHER2 as a new HER2 determination tool in breast cancer. We succeeded in showing a strong correlation between ISH ratio (HER2 gene to CEP17) and eHER2, and we demonstrated that $e H E R 2$ has the potential to replace the conventional HER2 examination methods. A larger scale study is needed to apply our method in clinical use.

\section{Declarations}

\section{Ethics approval}

This study was carried out with permissions from the University of Tokyo Hospital Ethics Committee (Approved No. 11031-(1)). All methods presented here were performed in accordance with the relevant guidelines and regulations approved by Faculty of Medicine, the University of Tokyo.

\section{Consent to participate}

Written informed consent was obtained from all participants.

\section{Consent for publication}

Patients signed informed consent regarding their data.

\section{Competing interests}

All authors of this work declare that they have no conflict of interest.

\section{Funding}

This work was supported by Basic Research Fund from the University of Tokyo.

\section{Authors' contributions}


K.O. and T.S. conceived the study. T.S., M.T. and Y.S. directed the study and supervised the research. K.O. collected tumor specimens. K.O. and T.S. confirmed histopathology findings and interpreted the clinical data. K.O. performed droplet digital PCR analyses. K.O. wrote the manuscript, with the assistance and final approval of all authors.

\section{Availability of data and material}

All data supporting the study are available on request. No proprietary materials except patient tissues were used.

\section{Acknowledgement}

We are grateful to Aiko Nishimoto, Kei Sakuma, and Tami Ohmi for their technical support. We thank the Edanz Group (www.edanzediting.com/ac) for editing a draft of this manuscript.

\section{References}

1. Moelans CB, de Weger RA, Van der Wall E, van Diest PJ. Current technologies for HER2 testing in breast cancer. Crit Rev Oncol Hematol. 2011;80:380-92.

2. Wolff AC, Hammond ME, Schwartz JN, Hagerty KL, Allred DC, Cote RJ. American Society of Clinical Oncology/College of American Pathologists guideline recommendations for human epidermal growth factor receptor 2 testing in breast cancer. J Clin Oncol. 2007; 25:118-45.

3. Otsuji K, Sasaki T, Tanaka A, Kunita A, Ikemura M, Matsusaka K, et al. Use of droplet digital PCR for quantitative and automatic analysis of the HER2 status in breast cancer patients. Breast Cancer Res Treat. 2017;162:11-8.

4. Hindson BJ, Ness KD, Masquelier DA, Belgrader P, Heredia NJ, Makarewicz AJ, et al. High-throughput droplet digital PCR system for absolute quantitation of DNA copy number. Anal Chem. 2011;83:860410.

5. Wolff AC, Hammond ME, Hicks DG, Dowsett M, McShane LM, Allison KH, et al. Recommendations for Human Epidermal Growth Factor Receptor 2 Testing in Breast Cancer: American Society of Clinical Oncology/College of American Pathologists Clinical Practice Guideline Update. J Clin Oncol. 2013;31:3997-4013.

6. Garcia-Murillas I, Lambros M, Turner NC. Determination of HER2 amplification status on tumour DNA by digital PCR. PLoS One. 2013;8:e83409.

7. Braun M, Kirsten R, Rupp NJ, Moch H, Fend F, Wernert N, et al. Quantification of protein expression in cells and cellular subcompartments on immunohistochemical sections using a computer supported image analysis system. Histol Histopathol. 2013;28:605-10. 
8. Nikolaishvilli-Feinberg N, Cohen SM, Midkiff B, Zhou Y, Olorvida M, Ibrahim JG, et al. Development of DNA damage response signaling biomarkers using automated, quantitative image analysis. $\mathrm{J}$ Histochem Cytochem. 2013;62:185-96.

9. Fisel P, Stühler V, Bedke J, Winter S, Rausch S, Hennenlotter J, et al. MCT4 surpasses the prognostic relevance of the ancillary protein CD147 in clear cell renal cell carcinoma. Oncotarget. 2015;6:3061527.

10. Wolff AC, Hammond MEH, Allison KH, Harvey BE, Mangu PB, Bartlett JMS, et al. Human Epidermal Growth Factor Receptor 2 Testing in Breast Cancer: American Society of Clinical Oncology/College of American Pathologists Clinical Practice Guideline Focused Update. J Clin Oncol. 2018;36(20):210522.

11. Wang Y, Tsang JYS, Cui Y, Cui J, Lin Y, Zhao S, et al. Robust and accurate digital measurement for HER2 amplification in HER2 equivocal breast cancer diagnosis. Sci Rep. 2017;7(1):6752.

12. Xu QQ, Pan B, Wang CJ, Zhou YD, Mao F, Lin Y, et al. HER2 amplification level is not a prognostic factor for HER2-positive breast cancer with trastuzumab-based adjuvant treatment: a systematic review and meta-analysis. Oncotarget. 2016;7:63571-82.

\section{Tables}

Due to technical limitations, table 1 and 2 is only available as a download in the Supplemental Files section.

\section{Figures}


(a)

Tissue with
only normal cells
$(\mathrm{TCR}[X]=0)$

\section{(c)}

Tissue with only normal cells (TCR $[X]=0$ )

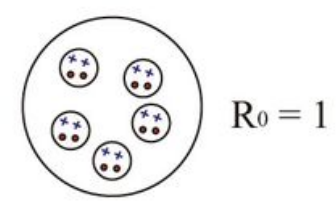

Tissue with both normal and tumor cells $(\operatorname{TCR}[X]=x)$

Tissue with only tumor cells (TCR $[X]=1$ )
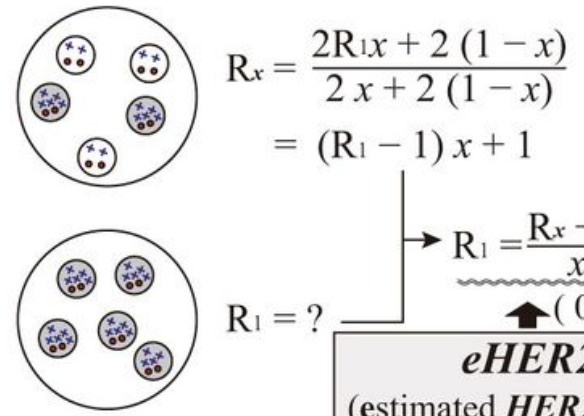

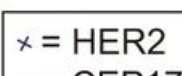

- = CEP17 (b)

$$
\text { ddPCR }
$$

ratio $[R]$

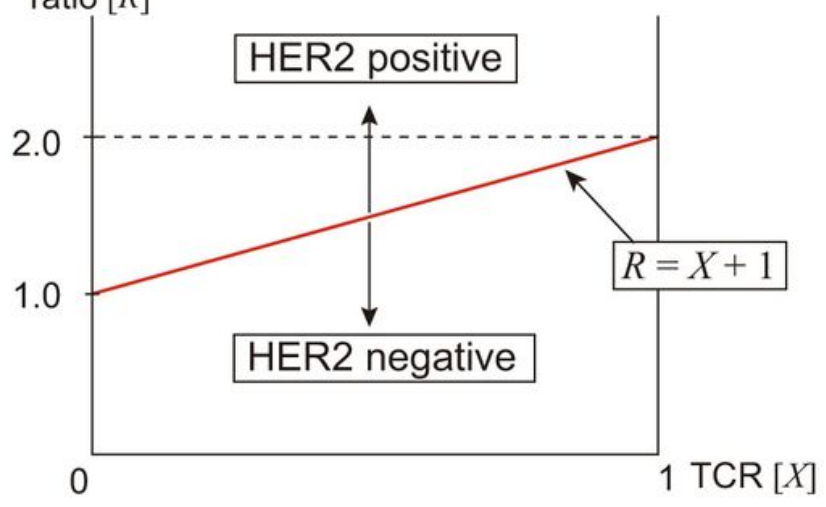

(d)

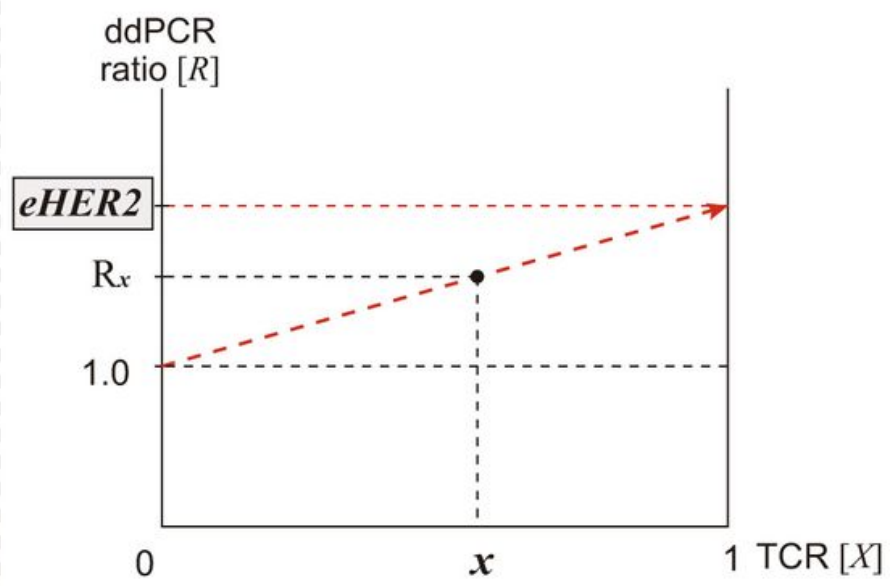

\section{Figure 1}

Concept of ddPCR-TCR method $(a, b)$ and how to calculate eHER2 $(c, d)$. (a) If there are exactly twice as many HER2 genes as CEP17 in a tumor cell, the HER2/CEP17 ratio obtained by ddPCR [Rx] can be expressed as $x+1$. (b) The ddPCR-TCR chart: a two-dimensional chart to determine HER2 gene amplification. The vertical axis represents the ddPCR ratio $[R](R>0)$ and the horizontal axis represents the TCR $[X](0<X \leq 1)$. When one of the samples is plotted above the theoretical cut-off line $R=x+1$, the cancer cells in this sample are considered to have HER2 gene amplification (HER2/CEP17 ratio over 2.0). (c) For a sample in which the HER2/CEP17 ratio in tumor cells (R1) is unknown but its ddPCR ratio [Rx] and TCR $[x]$ are successfully obtained, Rx can be expressed as "(R1 - 1) $x+1$ ". Thus, R1 can be calculated from the following formula: $(\mathrm{Rx}-1) / \mathrm{x}+1(0<\mathrm{x} \leq 1)$. We named this HER2/CEP17 ratio "R1" calculated from ddPCR ratio $[R x]$ and TCR $[x]$ as "eHER2". (d) A graphical concept of eHER2. Obtained $\operatorname{TCR}(x)$ bears a proportionate relationship to $(R x-1)$, so $(R x-1) / x$ is equal to $(R 1-1) / 1$. Hence eHER2 (= R1) can be expressed as $(R x-1) / x+1$. Also, eHER2 (=R1) can be calculated by plugging " $X=$ 1 " into $R=R x-1 / x^{\star} X+1$, a straight line connecting two points, $(X, R)=(0,1)$ and $(x, R x)$ on the graph. 
(a)

Tissue with
only normal cells
$(\mathrm{TCR}[X]=0)$

\section{(c)}

Tissue with only normal cells (TCR $[X]=0$ )

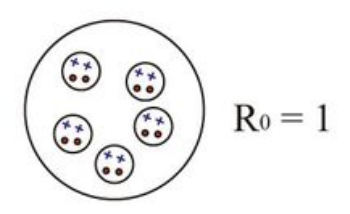

Tissue with both normal and tumor cells $(\operatorname{TCR}[X]=x)$

Tissue with only tumor cells (TCR $[X]=1$ )
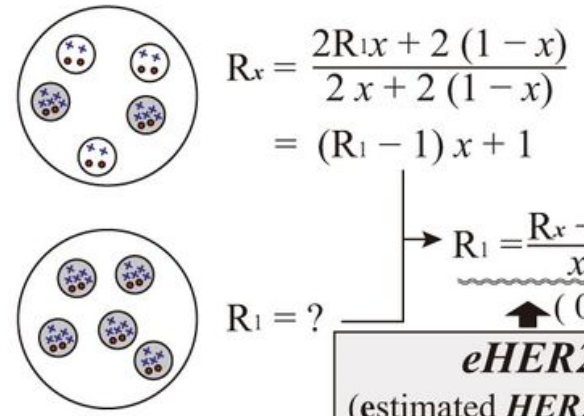

$x=$ HER2

$\bullet=$ CEP17 (b)

$$
\operatorname{ddPCR}
$$

ratio $[R]$

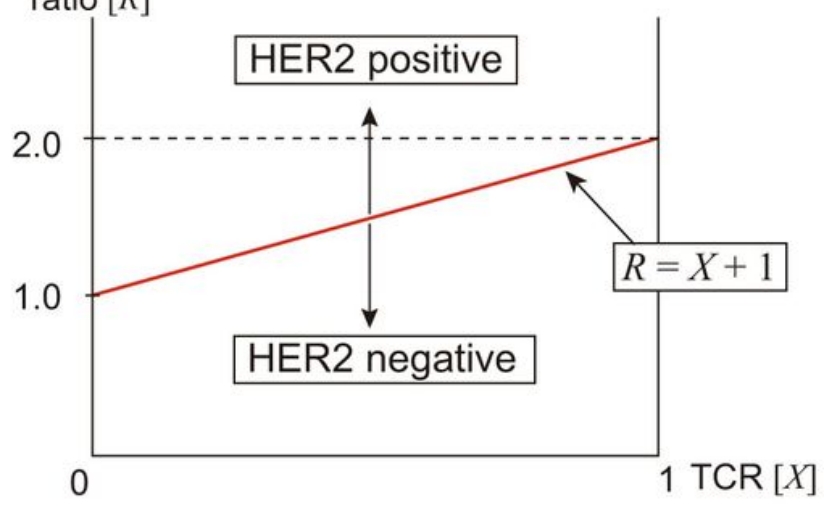

(d)

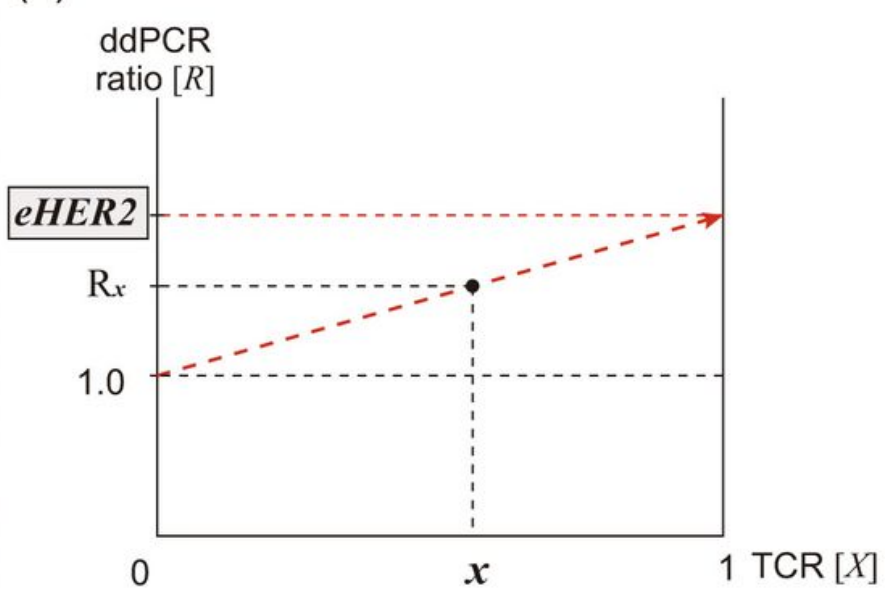

\section{Figure 1}

Concept of ddPCR-TCR method $(a, b)$ and how to calculate eHER2 $(c, d)$. (a) If there are exactly twice as many HER2 genes as CEP17 in a tumor cell, the HER2/CEP17 ratio obtained by ddPCR [Rx] can be expressed as $x+1$. (b) The ddPCR-TCR chart: a two-dimensional chart to determine HER2 gene amplification. The vertical axis represents the ddPCR ratio $[R](R>0)$ and the horizontal axis represents the TCR $[X](0<X \leq 1)$. When one of the samples is plotted above the theoretical cut-off line $R=X+1$, the cancer cells in this sample are considered to have HER2 gene amplification (HER2/CEP17 ratio over 2.0). (c) For a sample in which the HER2/CEP17 ratio in tumor cells (R1) is unknown but its ddPCR ratio [Rx] and TCR $[x]$ are successfully obtained, Rx can be expressed as "(R1-1) $x+1$ ". Thus, R1 can be calculated from the following formula: $(\mathrm{Rx}-1) / \mathrm{x}+1(0<\mathrm{x} \leq 1)$. We named this HER2/CEP17 ratio "R1" calculated from ddPCR ratio $[R x]$ and TCR $[x]$ as "eHER2". (d) A graphical concept of eHER2. Obtained TCR $(x)$ bears a proportionate relationship to $(R x-1)$, so $(R x-1) / x$ is equal to $(R 1-1) / 1$. Hence eHER2 (= R1) can be expressed as $(R x-1) / x+1$. Also, eHER2 (=R1) can be calculated by plugging " $X=$ 1 " into $R=R x-1 / x^{\star} X+1$, a straight line connecting two points, $(X, R)=(0,1)$ and $(x, R x)$ on the graph. 
(a)

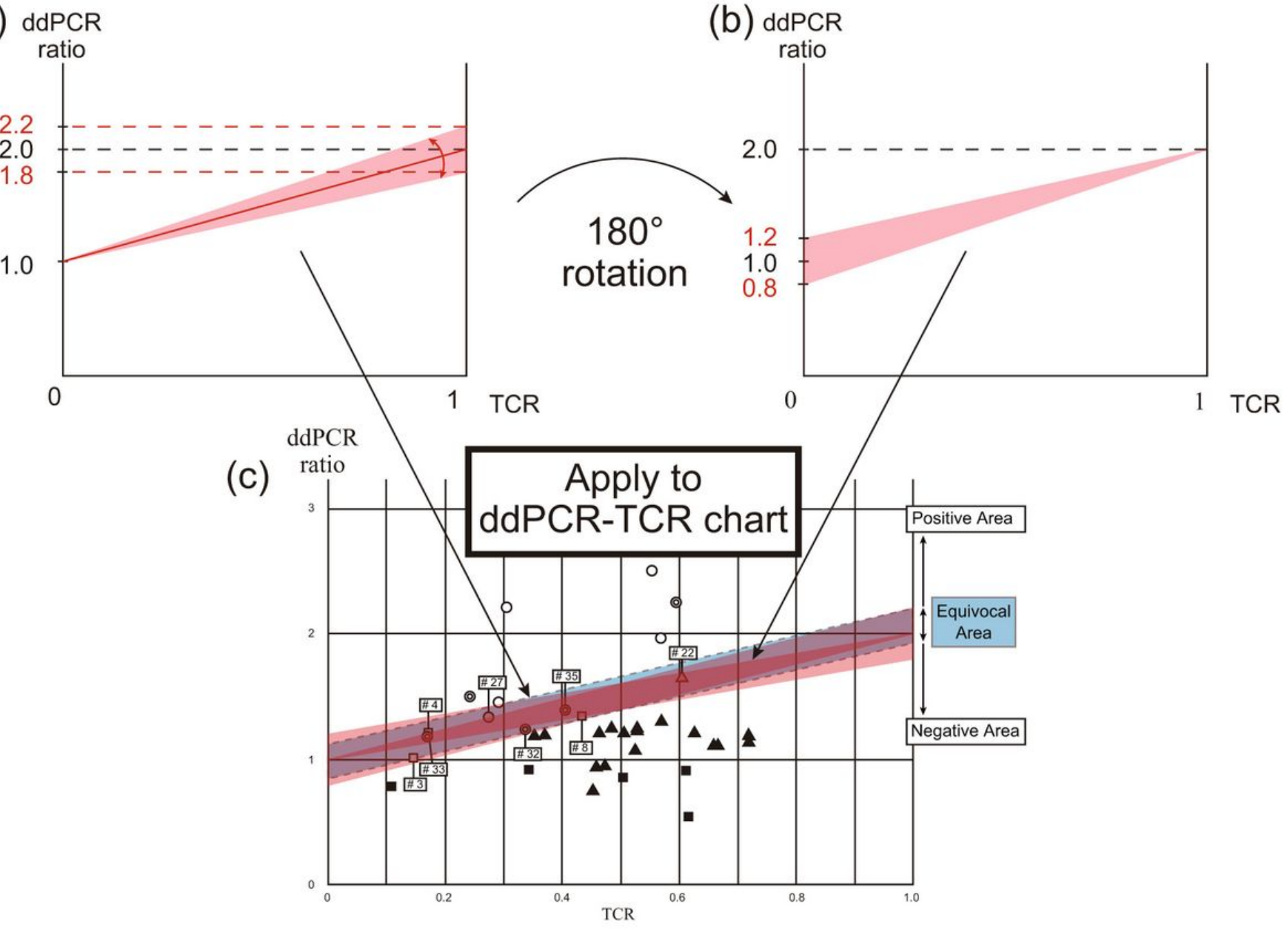

\section{Figure 2}

Newly designed equivocal ranges. (a) The range 1.8-2.2 was adopted as equivocal range of eHER2 based on the equivocal range for ISH assays of HER2 gene amplification in the former version of ASCO/CAP guidelines [2]. A sample with eHER2 between the range 1.8 to 2.2 , or whose $R x$ is between $0.8 x+1$ and $1.2 x+1$, has both possibility of HER2-gene twice amplified and not. (b) The equivocal area in (a) is rotated and fit on ddPCR-TCR chart. This second equivocal area indicates that the determination of HER2 status by calculating eHER2 can be misleading if a sample is plotted in it, or in other words, if Rx of the sample is between $1.2 x+0.8$ and $0.8 x+1.2$. (c) When applying these equivocal areas from $(a, b)$ on ddPCR-TCR chart with the clinical cases plotted [3], these areas almost overlap the previous equivocal area (shaded in blue). Also, cases within the previous equivocal area (cases 3, 4, 8, 22, 27, 32, 33 and 35 on Table 2) are all included in the newly combined equivocal areas. 
(a)

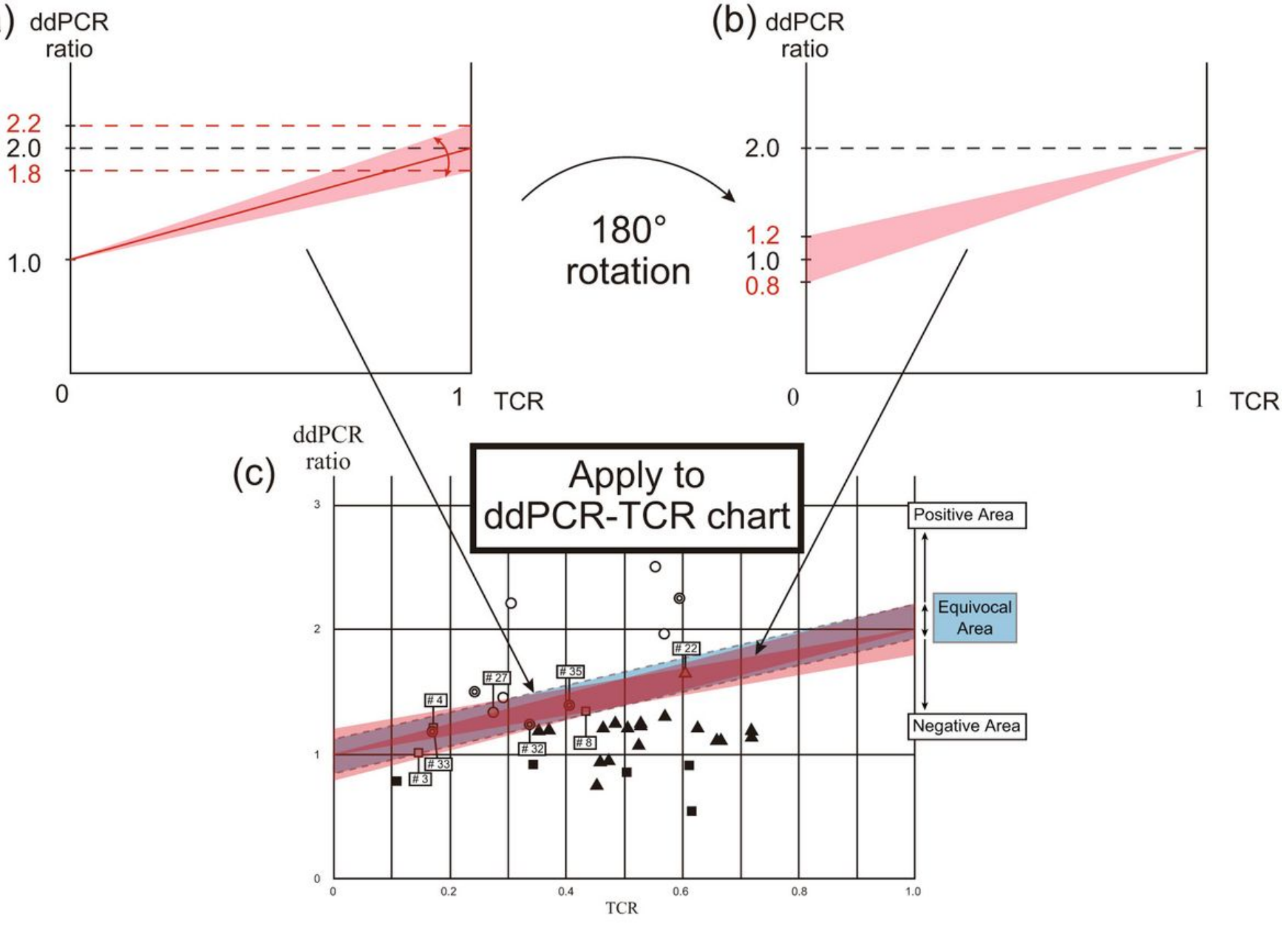

\section{Figure 2}

Newly designed equivocal ranges. (a) The range 1.8-2.2 was adopted as equivocal range of eHER2 based on the equivocal range for ISH assays of HER2 gene amplification in the former version of ASCO/CAP guidelines [2]. A sample with eHER2 between the range 1.8 to 2.2 , or whose Rx is between $0.8 x+1$ and $1.2 x+1$, has both possibility of HER2-gene twice amplified and not. (b) The equivocal area in (a) is rotated and fit on ddPCR-TCR chart. This second equivocal area indicates that the determination of HER2 status by calculating eHER2 can be misleading if a sample is plotted in it, or in other words, if Rx of the sample is between $1.2 x+0.8$ and $0.8 x+1.2$. (c) When applying these equivocal areas from $(a, b)$ on ddPCR-TCR chart with the clinical cases plotted [3], these areas almost overlap the previous equivocal area (shaded in blue). Also, cases within the previous equivocal area (cases 3, 4, 8, 22, 27, 32,33 and 35 on Table 2) are all included in the newly combined equivocal areas. 
a

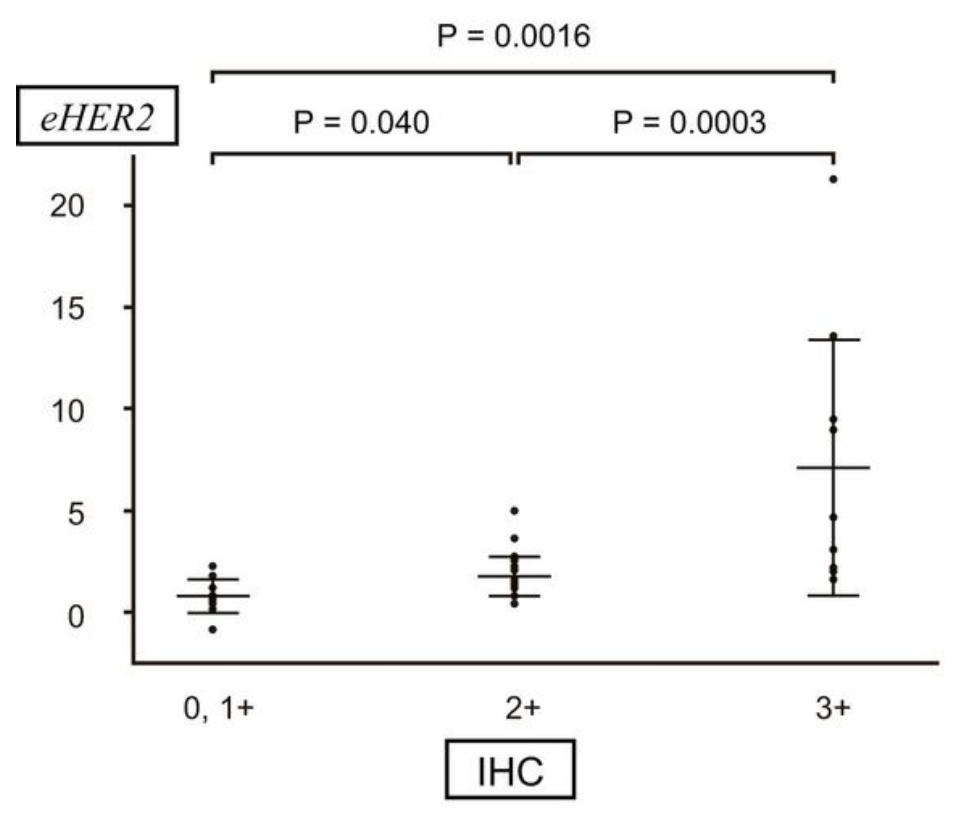

C

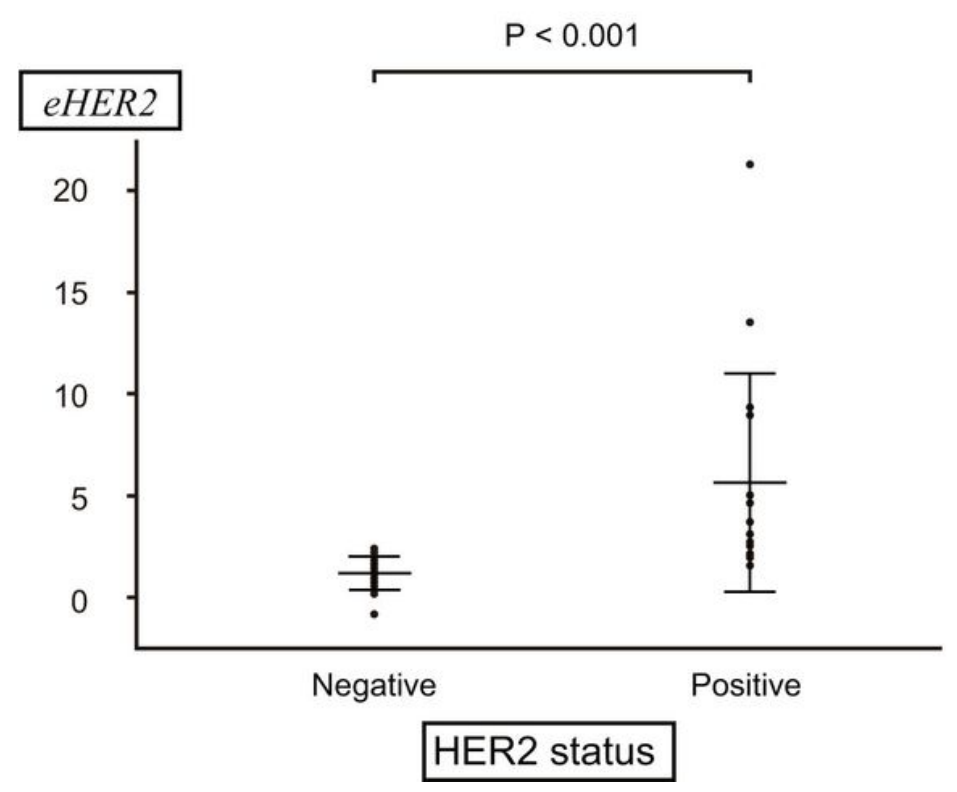

b

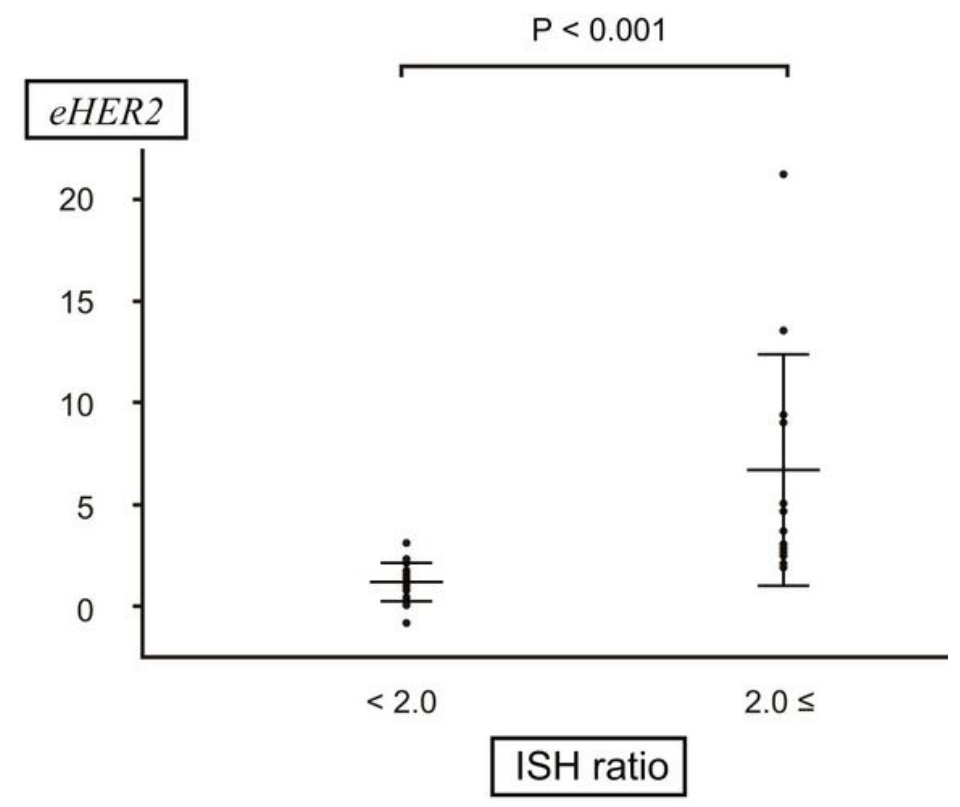

d

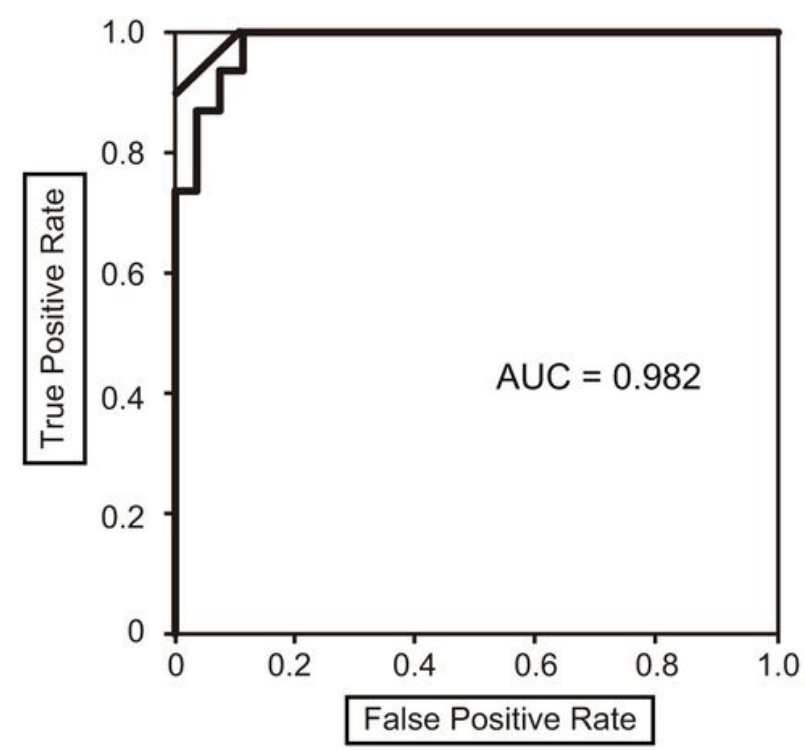

Figure 3

eHER2 among the development cohort. (a) Dot plot showing eHER2 with different IHC score. (b) Dot plot showing eHER2 with ISH ratio $\geq 2.0$ and $<2.0$ cases. (c) Dot plot showing eHER2 with HER2 positivity, based on ASCO/CAP guideline 2013 [5]. (d) ROC curve to determine the optimal cutoff. 
a

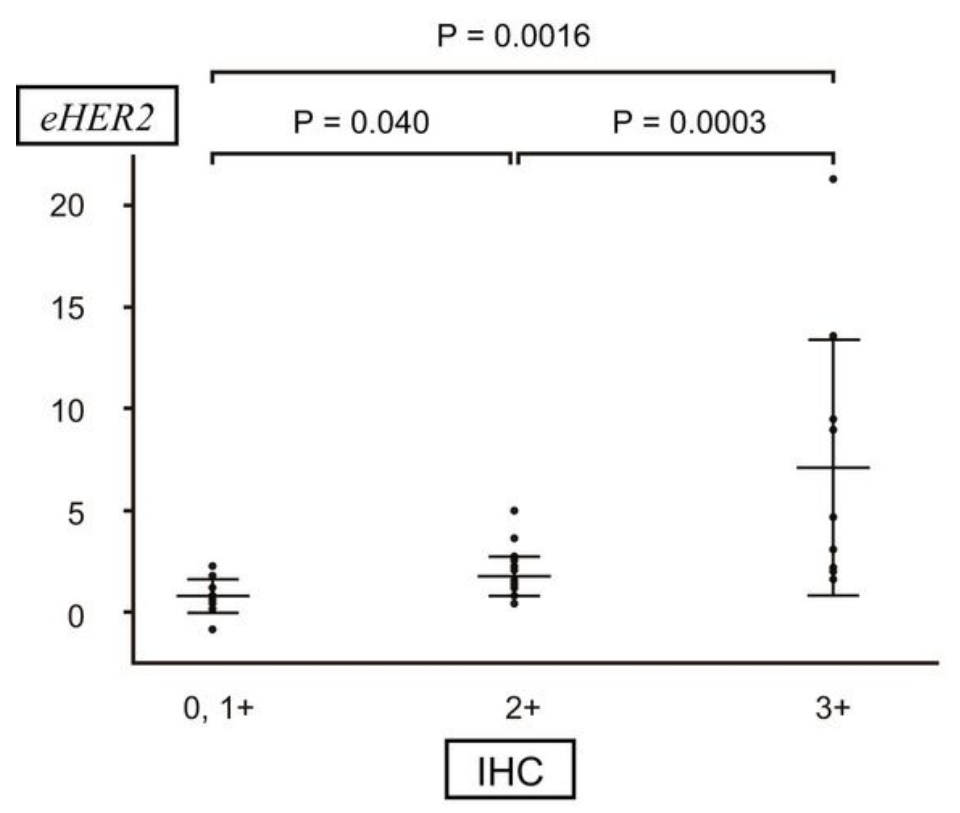

C

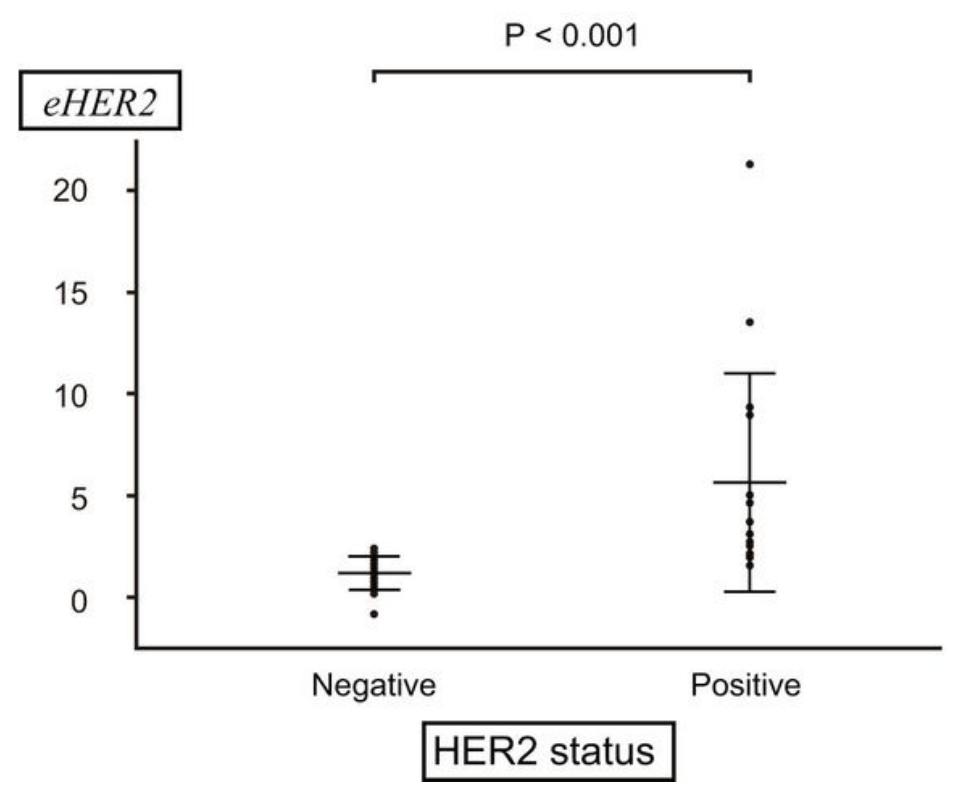

b

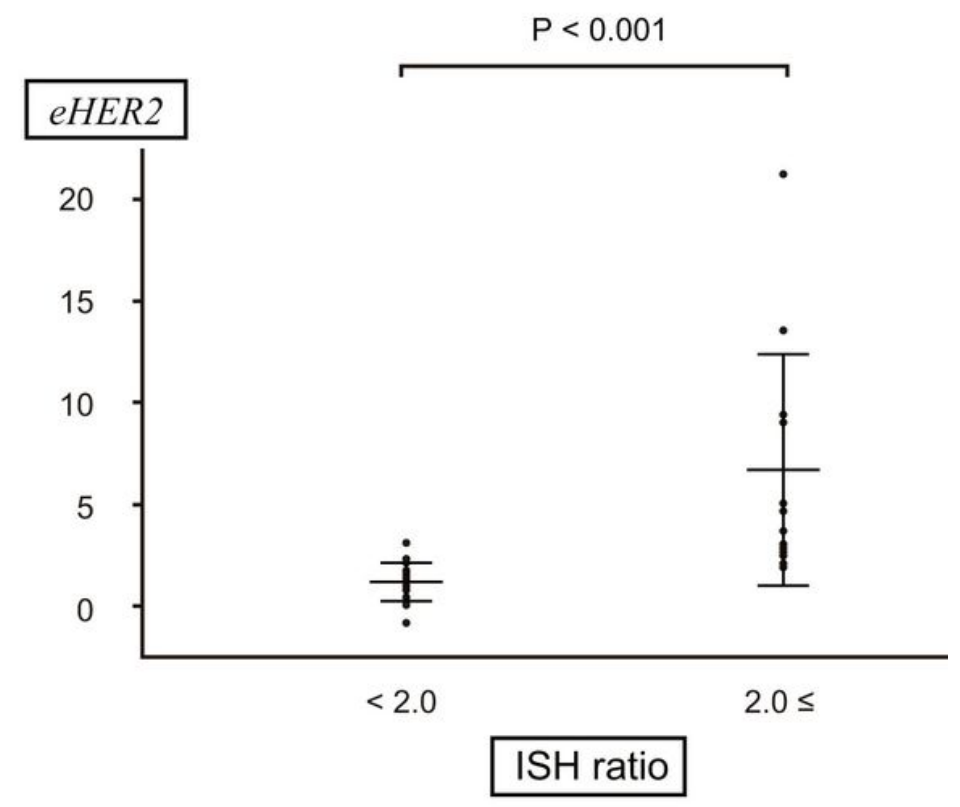

d

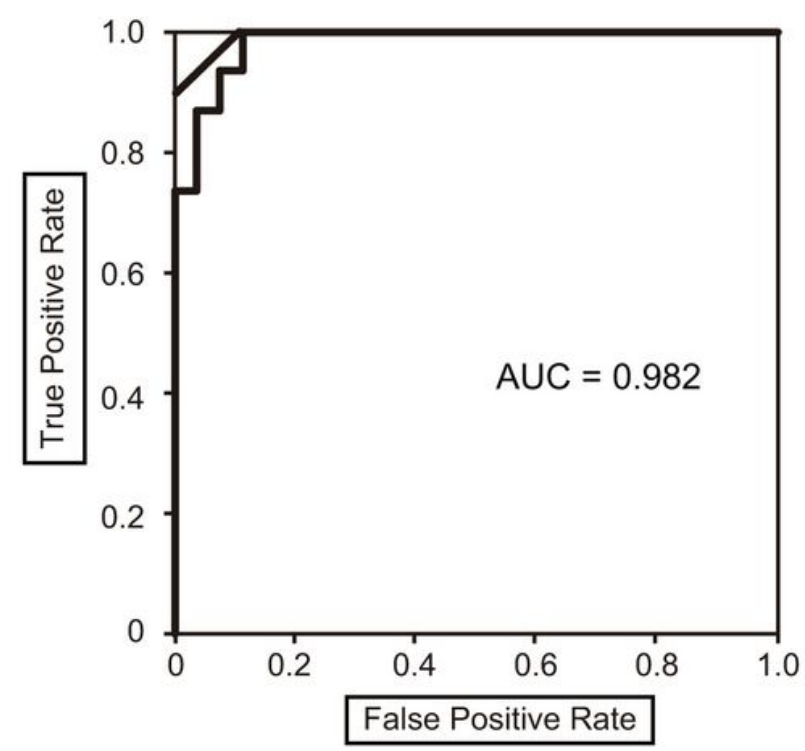

Figure 3

eHER2 among the development cohort. (a) Dot plot showing eHER2 with different IHC score. (b) Dot plot showing eHER2 with ISH ratio $\geq 2.0$ and $<2.0$ cases. (c) Dot plot showing eHER2 with HER2 positivity, based on ASCO/CAP guideline 2013 [5]. (d) ROC curve to determine the optimal cutoff. 


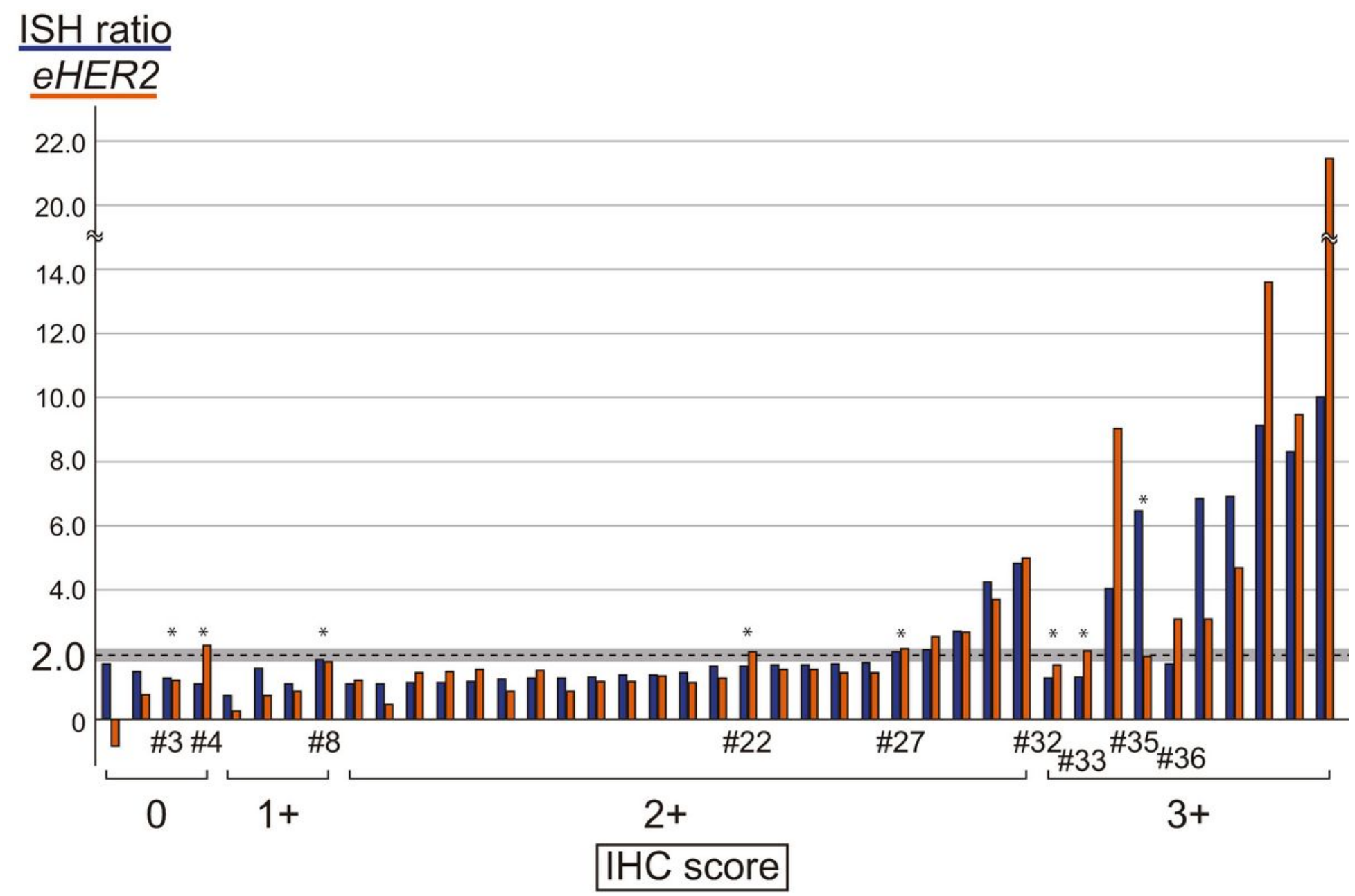

Figure 4

HER2/CEP17 ratio with ISH and eHER2 for clinical breast cancer cases in this study. The HER2/CEP17 ratio of ISH (blue bars) and eHER2 (orange bars) are indicated for each sample. Cases 1 to 41 are lined from left to right on the horizontal axis, and their IHC score according to ongoing ASCO/CAP guidelines [5] are indicated underneath the horizontal axis. The theoretical cut-off value of 2.0 is depicted as dotted line, and one of the equivocal ranges 1.8-2.2 is expressed as gray zone on the chart. Cases within either or both of two newly designed equivocal ranges are marked with asterisks above the bars, and their case numbers are indicated on the chart. All cases with an ISH ratio $\geq 2.0$ showed a high eHER2, at least over 1.8. There were two discrepant cases (cases 4 and 36) in which the ISH ratio were less than 1.8 but eHER2 were over 2.2. Of these, case 4 was included within the equivocal ranges, but case 36 was outside of the ranges. 


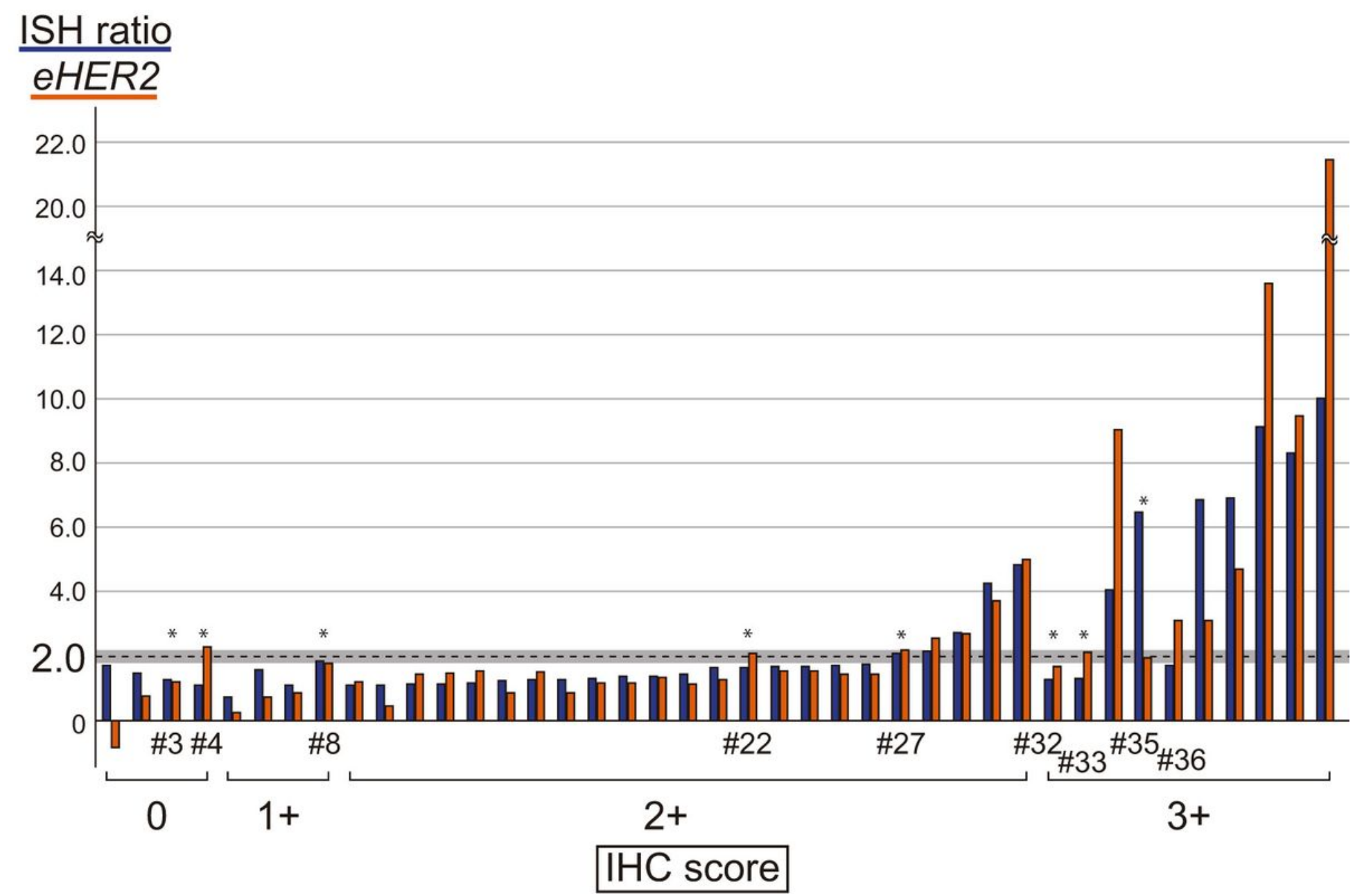

Figure 4

HER2/CEP17 ratio with ISH and eHER2 for clinical breast cancer cases in this study. The HER2/CEP17 ratio of ISH (blue bars) and eHER2 (orange bars) are indicated for each sample. Cases 1 to 41 are lined from left to right on the horizontal axis, and their IHC score according to ongoing ASCO/CAP guidelines [5] are indicated underneath the horizontal axis. The theoretical cut-off value of 2.0 is depicted as dotted line, and one of the equivocal ranges 1.8-2.2 is expressed as gray zone on the chart. Cases within either or both of two newly designed equivocal ranges are marked with asterisks above the bars, and their case numbers are indicated on the chart. All cases with an ISH ratio $\geq 2.0$ showed a high eHER2, at least over 1.8. There were two discrepant cases (cases 4 and 36) in which the ISH ratio were less than 1.8 but eHER2 were over 2.2. Of these, case 4 was included within the equivocal ranges, but case 36 was outside of the ranges.

\section{Supplementary Files}

This is a list of supplementary files associated with this preprint. Click to download.

- Table1.xls

- Table1.xls 
- Table2.xls

- Table2.xls

Page 20/20 